\title{
ADEQUAÇÃO DO TESTE DE GERMINAÇÃO PARA SEMENTES DE NABO FORRAGEIRO ${ }^{1}$
}

\author{
MARCELA CARLOTA NERY², MARIA LAENE MOREIRA DE CARVALHOㅇ, ANTONIO CARLOS FRAGA ${ }^{3}$
}

\begin{abstract}
RESUMO - O estabelecimento de condições ideais para condução do teste de germinação de sementes de nabo forrageiro é extremamente útil para avaliação da qualidade de lotes. Para definir a temperatura ótima e substrato ideal para a avaliação da germinação de sementes de nabo forrageiro, foram utilizadas sementes de diferentes lotes das cultivares CATI AL-1000 e IPR 116. A semeadura foi efetuada nos substratos papel e areia, e as sementes, mantidas em seis regimes de temperatura constante $\left(15^{\circ} \mathrm{C} ; 20^{\circ} \mathrm{C} ; 25^{\circ} \mathrm{C} ; 30^{\circ} \mathrm{C} ; 35^{\circ} \mathrm{C}\right)$ e um alternado $\left(20^{\circ} \mathrm{C}-30^{\circ} \mathrm{C}\right)$. A qualidade das sementes de nabo forrageiro foi avaliada pelas determinações do grau de umidade, teste de germinação, primeira contagem da germinação, índice de velocidade de germinação, emergência, estande inicial, índice de velocidade de emergência e teste de sanidade. A temperatura de $20^{\circ} \mathrm{C}-30^{\circ} \mathrm{C}$ e o substrato areia são condições adequadas para a realização do teste de germinação das sementes de nabo forrageiro das cultivares CATI AL-1000 e IPR 116.
\end{abstract}

Termo para indexação: Raphanus sativus, substrato, temperatura.

\section{ADEQUATION OF THE OIL RADISH SEEDS GERMINATION TEST}

\begin{abstract}
The determination of the ideal conditions to carry out the germination test on fodder radish seeds is extremely useful to evaluate the quality of seed lots. To determine the appropriate temperature and ideal substrate in the germination evaluation of fodder radish. Seeds, from different lots of the CATI AL-1000 and IPR 116 A cultivars were used. They were sown on paper and sand substrates, and the seeds were maintained under six constant temperature regimens $\left(15^{\circ} \mathrm{C} ; 20^{\circ} \mathrm{C}\right.$; $\left.25^{\circ} \mathrm{C} ; 30^{\circ} \mathrm{C} ; 35^{\circ} \mathrm{C}\right)$ and one alternating regimen $\left(20^{\circ} \mathrm{C}-30^{\circ} \mathrm{C}\right)$. The fodder radish seed quality was evaluated by determining the moisture content, germination test, first germination count, germination speed index and health quality. The temperature of $20^{\circ} \mathrm{C}-30^{\circ} \mathrm{C}$ and the sand substrate were the appropriate conditions to carry out the germination test on fodder radish seeds of the CATI AL-1000 and IPR 116 cultivars.
\end{abstract}

Index terms: Raphanus sativus, substrate, temperature.

${ }^{1}$ Submetido em 20/09/2008. Aceito para publicação, 11/03/2009. Parte da Tese de Doutorado do primeiro autor apresentado a UFLA.

${ }^{2}$ Eng. Agr.; Dr., Departamento de Agricultura, UFLA, Caixa Postal 3037,
Lavras, MG, CEP: 37200-000 - e-mail: nery.marcela@gmail.com,

${ }^{3}$ Eng. Agr.; Dr; Professor Titular, Departamento de Agricultura, UFLA. Caixa Postal 3037, Lavras, MG. CEP: 37200-000. 


\section{INTRODUÇÃO}

O nabo forrageiro, Raphanus sativus L. var. oleiferus Metzg., por apresentar em suas sementes consideráveis teores de óleo com relativa facilidade de extração, além de possuir potencial produtivo em épocas de pouca utilização ou pousio das terras agricultáveis, poderá se tornar matéria-prima de substancial interesse aos produtores rurais para produção de óleo no Programa do Biodiesel (Silva, 2007).

É uma planta originária da Ásia Oriental e Europa, pertencente à família Brassicaceae, da ordem Caparales, com ciclo anual de inverno (Muzilli, 2002). O fruto é uma síliqua indeiscente, de $3 \mathrm{~cm}$ a $5 \mathrm{~cm}$ de comprimento, contendo de 2 a 10 sementes, com massa de 1000 grãos variando de 6 g a 14 g, com média de 11 g (Derpsch e Calegari, 1992). Apesar do nabo forrageiro se reproduzir via sementes, pouco se sabe sobre a produção e qualidade das mesmas. Ainda não existe metodologia adequada para realização do teste de germinação capazes de permitir obter informações sobre o potencial máximo de germinação de lotes de sementes das cultivares disponíveis no mercado nacional, CATI AL-1000 e IRP 116.

O conhecimento das condições ideais para a germinação das sementes de uma determinada espécie é de fundamental importância, principalmente, pelas respostas diferenciadas que a semente pode expressar em função de diversos fatores, como viabilidade, dormência (Finch-Savage e Metzger, 2006), condições de ambiente (Chen et al., 2005), envolvendo água, luz (Probert et al., 1986), temperatura (Simpson e Dean, 2002), oxigênio e ausência de agentes patogênicos (Koger et al. 2004).

Dentre os fatores do ambiente que afetam a germinação das sementes, a temperatura exerce influência na velocidade e na porcentagem final de germinação (Bewley e Black, 1994). A temperatura definida como ótima é aquela na qual a semente expressa seu potencial máximo de germinação no menor tempo possível e as temperaturas máxima e mínima caracterizam pontos críticos, acima e abaixo dos quais não ocorre germinação. A temperatura máxima aumenta a velocidade de germinação, mas somente as sementes mais vigorosas conseguem germinar, o que determina uma redução na porcentagem de germinação. Já a temperatura mínima reduz a velocidade de germinação e altera a uniformidade de emergência (Marshall e Squire, 2000). Além disso, devido ao maior tempo necessário para as sementes germinarem, estas ficam expostas ao ataque de patógenos (Szopińska et al., 2007).

Vários são os trabalhos com sementes da família
Brassicaceae que buscam estabelecer a temperatura mais adequada para a condução do teste de germinação. Para Brassica chinensis L., couve-da-malásia, o regime de temperatura recomendado para germinação é o alternado $20^{\circ} \mathrm{C}-30^{\circ} \mathrm{C}$ (Ellis et al., 1989). Já para B. napus oleifera $\mathrm{L}$. ou $B$. napus L., colza, há relatos de diferentes temperaturas ótimas para a germinação das sementes, as quais variam de $10^{\circ} \mathrm{C}$ a $30^{\circ} \mathrm{C}$ (Squire et al., 1997). Tokumasu e Kakihara (1990) descreveram que a temperatura mínima de $23^{\circ} \mathrm{C}$ e a máxima de $27^{\circ} \mathrm{C}$ são as recomendadas para a germinação da cultura da colza. A temperatura alternada é recomendada para $B$. tournefortii Gouan., mostarda, em que a temperatura ótima é de $12^{\circ} \mathrm{C}-20^{\circ} \mathrm{C}$ (Chauhan et al., 2006). Para Lesquerella fendleri (Gray) Wats., lesquerella, também da família Brassicaceae, a germinação das sementes ocorre mais rapidamente a partir de $18^{\circ} \mathrm{C}$ atingindo valores máximos a $22^{\circ} \mathrm{C}$ (Adam et al., 2007). A temperatura de $21^{\circ} \mathrm{C}$ é a recomendada para Cuphea sp., flor-de-santo-antônio, uma espécie oleaginosa (Berti et al., 2007).

Além da temperatura, o substrato utilizado no teste de germinação, também afeta os resultados. O substrato, em geral, tem como principal função dar sustentação às sementes (Aguiar et al., 1993), além de influenciar na aeração, capacidade de retenção de água e grau de infestação de patógenos nas sementes. Em função do tamanho e das exigências ecofisiológicas das sementes quanto à umidade $\mathrm{e}$ luz, cada substrato é utilizado de maneira que ofereça maior praticidade nas contagens e avaliação das plântulas (Oliveira et al., 2001). Fanti e Perez (1999) descrevem que na escolha do material para o substrato deve ser levado em consideração o tamanho das sementes, sua exigência com relação à umidade, sensibilidade ou não à luz e, ainda, a facilidade que este oferece para o desenvolvimento das plântulas. $\mathrm{O}$ substrato utilizado no teste de germinação é muito importante para obtenção de resultados confiáveis, em vista, sobretudo, da grande variação que existe entre as espécies com relação ao substrato mais adequado (Alvino e Rayol, 2007). Segundo Tobe et al. (2005), a utilização do substrato areia no teste de germinação deve ser estudada, pois a umidade do substrato varia dependendo das condições do ambiente, afetando o crescimento das plântulas.

Para Raphanus sativus var. oleiferus, não há ainda recomendações para a metodologia do teste de germinação nas Regras para Análise de Sementes, RAS (Brasil, 1992). A metodologia que vem sendo utilizada é a mesma empregada para Raphanus ou para Brassicas. Para esses gêneros as RAS prescrevem que as sementes deve ser pré-resfriadas a $5^{\circ} \mathrm{C}$ ou $10^{\circ} \mathrm{C}$ por 7 dias, após o que no teste de germinação pode 
ser utilizado o substrato papel, entre papel ou entre areia, temperaturas de $20^{\circ} \mathrm{C}-30^{\circ} \mathrm{C}$ alternada ou $20^{\circ} \mathrm{C}$ constante. Já o Commercial Agriculture Development (2006) recomenda que o teste de germinação de sementes de Raphanus seja realizado a temperatura de $21^{\circ} \mathrm{C}$ também por período de 7 dias.

A qualidade sanitária das sementes é outro aspecto a ser considerado, visando à adequação do teste de germinação em brássicas, pois o grau de infecção por patógenos pode variar entre os lotes e os microrganismos podem proliferar de maneira diferenciada de um substrato para outro, interferindo na germinação e no estabelecimento de plântulas (Pereira et al., 2005). Dentre os patógenos mais encontrados em sementes de brássicas, o gênero Alternaria spp., agente da mancha-foliar nas silíquias, é um dos principais microrganismos que podem reduzir a germinação por seus danos às plântulas e ainda reduzir em até $35 \%$ o teor de óleo nas sementes (Humpherson-Jones, 1992). Shrestha et al. (2000) encontraram Alternaria brassicae no tegumento das sementes de canola (B. campestris L. var. toria) e mostarda (B. juncea L.).

Objetivou-se, com esta pesquisa, adequar a metodologia do teste de germinação para sementes de nabo forrageiro, visando definir a temperatura ótima e o substrato ideal para a avaliação da qualidade de sementes.

\section{MATERIAL E MÉTODOS}

Sementes de nabo forrageiro das cultivares CATI AL1000 (CATI) e IPR 116 (IAPAR), produzidas nas safras de 2004 e 2005, fornecidas pela Pesagro, RJ, foram submetidas às seguintes determinações e testes:

$\mathrm{O}$ grau de umidade foi determinado pelo método de estufa a $105 \pm 3^{\circ} \mathrm{C}$ por 24 horas (Brasil, 1992), com 2 repetições de $10 \mathrm{~g}$ de sementes, sendo os resultados expressos em porcentagem de peso úmido.

Para adequação do teste de germinação a semeadura foi realizada sobre substrato papel mata-borrão em caixas acrílicas do tipo gerbox, umedecido com água destilada em quantidade equivalente a 2,0 vezes o peso do substrato seco. Foi feita também a semeadura sobre areia, lavada e autoclavada, umedecida a $60 \%$ da capacidade de campo, sendo esta umidade definida em pré-teste, testando $40 \%, 50 \%$, $60 \%$ e $70 \%$ da capacidade de campo. As caixas de gerbox com sementes foram mantidas em mesa termogradiente, regulada nas temperaturas de $15^{\circ} \mathrm{C} ; 20^{\circ} \mathrm{C} ; 25^{\circ} \mathrm{C} ; 30^{\circ} \mathrm{C}$ e $35^{\circ} \mathrm{C}$ $\pm 2^{\circ} \mathrm{C}$, sob luz contínua e em câmara do tipo B.O.D. regulada à temperatura alternada de $20^{\circ} \mathrm{C}-30^{\circ} \mathrm{C}$, com fotoperíodo de
12 horas. Os resultados foram expressos em porcentagem de plântulas normais computadas diariamente para construção da curva de germinação acumulada e definição da data ideal para a primeira e última contagem do teste. A primeira contagem foi realizada no $3^{\circ}$ dia após a semeadura e a contagem final no $7^{\circ}$ dia. As sementes dormentes, ao final do teste, foram submetidas ao teste de tetrazólio, em que as sementes foram embebidas entre papel por 6 horas, seguida de corte longitudinal e imersão em solução $0,5 \%$ de tetrazólio, por 3 horas a $25^{\circ} \mathrm{C}$. O índice de velocidade de germinação foi calculado segundo a fórmula proposta por Maguire (1962), computando-se o número de sementes protrundidas a partir da emissão de $1 \mathrm{~mm}$ de radícula.

A emergência foi realizada sob condições controladas em câmara do tipo B.O.D. à $20^{\circ} \mathrm{C}$, sob luz constante e a semeadura, realizada em substrato solo e areia na proporção 1:2 em bandejas plásticas. A umidade do substrato foi ajustada para $60 \%$ da capacidade de retenção. Foram realizadas avaliações diárias, computando-se o estande inicial ao $3^{\circ}$ dia e número de plântulas emergidas até a estabilização, que totalizou 9 dias para cultivar CATI AL-1000 e 6 dias para IPR 116. O índice de velocidade de emergência (IVE) foi determinado segundo fórmula proposta por Maguire (1962).

Foi realizado o teste de sanidade das sementes de nabo forrageiro pelo método do papel de filtro ou blotter test modificado, com o uso de 2,4-D e congelamento, utilizandose 200 sementes, divididas em 8 repetições de 25 sementes dispostas em placas de Petri sobre três folhas de papel de filtro embebidas com água destilada, 2,4-D e ágar. As placas foram mantidas a temperatura de $-8^{\circ} \mathrm{C}$ por 1 dia e depois incubadas em câmara tipo B.O.D. a $22^{\circ} \mathrm{C}-25^{\circ} \mathrm{C}$, com fotoperíodo de 12 horas, por 5 dias. Foi avaliada a presença de fungos nas sementes, com auxílio de lupa e microscópico estereoscópico.

O delineamento utilizado foi o inteiramente casualizado, com quatro repetições de 50 sementes, em esquema fatorial $6 \times 2$ (6 regimes de temperaturas e 2 substratos) para cada cultivar. Os dados, previamente submetidos aos testes de normalidade dos resíduos e homocedasticidade das variâncias, foram submetidos à análise de variância e as médias comparadas entre si pelo teste de Tukey, a 5\% de probabilidade e transformadas em $\sqrt{x}$. As análises estatísticas foram realizadas com o auxílio do programa estatístico SISVAR ${ }^{\circledR}$ (Ferreira, 2000).

\section{RESULTADOS E DISCUSSÃO}

A umidade das sementes por ocasião da realização dos 
testes era de 5\% para o lote de 2004 e $6 \%$ para os lote de 2005 da cultivar CATI AL-1000, sendo esta a umidade de equilíbrio característica de espécies oleaginosas em temperatura ambiente de $25^{\circ} \mathrm{C}$ e umidade relativa do ar de $50 \%$ (Burrel et al., 1980).

Pela caracterização do perfil dos lotes da cultivar CATI AL -1000 (Tabela 1) observaram-se valores superiores no estande inicial, emergência e IVE para o lote produzido em 2005.

TABELA 1. Valores médios de estande inicial - EI (\%), emergência - E (\%) e índice de velocidade de emergência de plântulas de nabo forrageiro, cultivar CATI AL-1000, lotes de 2004 e 2005.

\begin{tabular}{lccc}
\hline \multicolumn{1}{c}{ Lotes } & EI $(\%)$ & E $(\%)$ & IVE \\
\hline 2004 & $23 \mathrm{~b}$ & $31 \mathrm{~b}$ & $8,98 \mathrm{~b}$ \\
2005 & $51 \mathrm{a}$ & $59 \mathrm{a}$ & $22,98 \mathrm{a}$ \\
\hline $\mathrm{CV}(\%)$ & 16,56 & 14,88 & 7,22 \\
\hline
\end{tabular}

Médias seguidas pela mesma letra, na coluna, não diferem entre si pelo teste de Tukey, a $5 \%$.

Apesar da superioridade do lote produzido em $2005 \mathrm{em}$ relação ao de 2004, a emergência de plântulas foi de apenas $59 \%$. Um dos aspectos que pode ter afetado a emergência é a qualidade sanitária das sementes de nabo forrageiro (Tabela 2). Observou-se elevada porcentagem de incidência do fungo Aspergillus flavus para o lote de 2004 e de Alternaria alternata, Aspergillus niger e Penicillium sp. para o lote de 2005. De acordo com Maude e Humpherson-Jones (1980) sementes de brássicas infectadas por Alternaria brassicae e A. brassicicola perdem sua viabilidade rapidamente.

TABELA2 . Porcentagem (\%) dos fungos Aspergilus flavus, Aspergilus niger, Penicilium, Alternaria alternata em sementes de nabo forrageiro, cultivar CATI AL-1000, lotes de 2004 e 2005.

\begin{tabular}{lcccc}
\hline Lotes & $\begin{array}{c}\text { Aspergillus } \\
\text { flavus } \\
(\%)\end{array}$ & $\begin{array}{c}\text { Aspergillus } \\
\text { niger } \\
(\%)\end{array}$ & $\begin{array}{c}\text { Penicilliu } \\
\text { m spp. } \\
(\%)\end{array}$ & $\begin{array}{c}\text { Alternaria } \\
\text { alternata } \\
(\%)\end{array}$ \\
\hline 2004 & $75 \mathrm{a}$ & $3 \mathrm{a}$ & $1 \mathrm{a}$ & $1 \mathrm{~b}$ \\
2005 & $8 \mathrm{~b}$ & $6 \mathrm{a}$ & $3 \mathrm{a}$ & $52 \mathrm{a}$ \\
\hline $\mathrm{CV}(\%)$ & 30,44 & 76,93 & 158,51 & 43,20 \\
\hline
\end{tabular}

Médias seguidas pela mesma letra, na coluna, não diferem entre si pelo teste de Tukey, a 5\%.
Também para cultivar IPR 116, o grau de umidade das sementes era de 5\% para ambos os lotes. Observa-se para o lote produzido em 2004, superioridade de resultados de estande inicial, emergência e índice de velocidade de emergência em relação ao lote de 2005 (Tabela 3).

TABELA 3. Valores médios de estande inicial - EI (\%), emergência - E (\%) e índice de velocidade de emergência - IVE de plântulas de nabo forrageiro, cultivar IPR 116, lotes de 2004 e 2005.

\begin{tabular}{cccc}
\hline Lotes & EI $(\%)$ & E (\%) & IVE \\
\hline 2004 & $38 \mathrm{a}$ & $46 \mathrm{a}$ & $11,24 \mathrm{a}$ \\
2005 & $9 \mathrm{~b}$ & $12 \mathrm{~b}$ & $6,38 \mathrm{a}$ \\
\hline $\mathrm{CV}(\%)$ & 40,28 & 38,25 & 23,50 \\
\hline
\end{tabular}

Médias seguidas pela mesma letra, na coluna, não diferem entre si pelo teste de Tukey, a $5 \%$.

Para cultivar IPR 116, porcentagem superior de incidência dos fungos Aspergillus flavus, Alternaria alternata e Penicillium sp. foi observada no lote de 2004 em relação ao lote de 2005 (Tabela 4). Alto nível de incidência de Aspergillus spp. e Penicillium spp. também foi observado infectando sementes de canola (Pronyk et al., 2006).

TABELA 4. Porcentagem(\%) dos fungos Aspergillusflavus, Aspergillus niger, Penicillium, Alternaria alternata em sementes de nabo forrageiro, cultivar IPR 116, lotes de 2004 e 2005.

Aspergillus Aspergillus Penicillium Alternaria Lotes flavus (\%) Níger (\%) sp. (\%) alternata

\begin{tabular}{lrcrc} 
& & & & $(\%)$ \\
\hline 2004 & $42 \mathrm{a}$ & $1 \mathrm{a}$ & $11 \mathrm{a}$ & $14 \mathrm{a}$ \\
2005 & $6 \mathrm{~b}$ & $2 \mathrm{a}$ & $0 \mathrm{~b}$ & $12 \mathrm{a}$ \\
\hline $\mathrm{CV}(\%)$ & 29,80 & 152,95 & 133,06 & 45,93 \\
\hline
\end{tabular}

Médias seguidas pela mesma letra, na coluna, não diferem entre si pelo teste de Tukey, a $5 \%$.

Observa-se, na Tabela 5, a superioridade na porcentagem de plântulas normais na primeira contagem da germinação para a cultivar CATI AL-1000, a germinação na primeira contagem de ambos os lotes em areia foi superior à obtida 
em papel, exceto na temperatura de $20^{\circ} \mathrm{C}-30^{\circ} \mathrm{C}$ onde não foram observadas diferenças significativas. No lote 2004, em substrato papel, observaram-se maiores valores na primeira contagem da germinação a $20^{\circ} \mathrm{C}-30^{\circ} \mathrm{C}$ Já em substrato areia, maior germinação foi constatada na temperatura de $15^{\circ} \mathrm{C}$, apesar dessa temperatura não diferir das demais.

TABELA 5. Germinação na primeira contagem (\%) de sementes de nabo forrageiro das cultivares CATI AL-1000 e IPR 116 lotes de 2004 e 2005, em diferentes temperaturas (T) e substratos sobre papel (SP) e sobre areia.

\begin{tabular}{|c|c|c|c|c|c|c|c|c|}
\hline \multirow{3}{*}{$\mathrm{T}\left({ }^{\circ} \mathrm{C}\right)$} & \multicolumn{4}{|c|}{ CATI AL-1000 } & \multicolumn{4}{|c|}{ IPR 116} \\
\hline & \multicolumn{2}{|c|}{2004} & \multicolumn{2}{|c|}{2005} & \multicolumn{2}{|c|}{2004} & \multicolumn{2}{|r|}{2005} \\
\hline & SP & Areia & SP & Areia & SP & Areia & SP & Areia \\
\hline 15 & $28 \mathrm{~b} \mathrm{~B}$ & 66 a A & $78 \mathrm{~b} \mathrm{~B}$ & 92 a A & $23 \mathrm{~b} \mathrm{AB}$ & 40 a B & $17 \mathrm{~b} \mathrm{~A}$ & 53 a A \\
\hline 20 & $41 \mathrm{~b} \mathrm{AB}$ & 58 a $\mathrm{AB}$ & $82 \mathrm{~b} \mathrm{AB}$ & 94 a A & $26 \mathrm{~b} \mathrm{AB}$ & 52 a $A B$ & 30 a $\mathrm{A}$ & 48 a A \\
\hline 25 & 39 b B & 56 a $\mathrm{AB}$ & 77 b B & 98 a A & 19 b B & $57 \mathrm{a} A B$ & $26 \mathrm{~b} \mathrm{~A}$ & 59 a A \\
\hline 30 & $11 \mathrm{~b} \mathrm{C}$ & 56 a $\mathrm{AB}$ & $36 \mathrm{~b} \mathrm{C}$ & 90 a A & $21 \mathrm{~b} \mathrm{~B}$ & 53 a $\mathrm{AB}$ & $11 \mathrm{~b} \mathrm{~A}$ & 59 a A \\
\hline 35 & $6 \mathrm{~b} \mathrm{C}$ & 49 a B & $0 \mathrm{~b} \mathrm{D}$ & 95 a A & $5 \mathrm{~b} \mathrm{~B}$ & 54 a $\mathrm{AB}$ & $14 \mathrm{~b} \mathrm{~A}$ & 72 a A \\
\hline $20-30$ & 53 a A & 60 a $\mathrm{AB}$ & 91 a A & 95 a A & $44 \mathrm{~b} \mathrm{~A}$ & 65 a A & $25 \mathrm{~b} \mathrm{~A}$ & 74 a A \\
\hline Média & $30 \mathrm{~b}$ & $58 \mathrm{a}$ & $61 \mathrm{~b}$ & $94 \mathrm{a}$ & $23 \mathrm{~b}$ & $54 \mathrm{a}$ & $21 \mathrm{~b}$ & $61 \mathrm{a}$ \\
\hline $\mathrm{CV}(\%)$ & \multicolumn{2}{|c|}{16,05} & \multicolumn{2}{|c|}{4,41} & \multicolumn{2}{|c|}{20,43} & \multicolumn{2}{|c|}{27,32} \\
\hline $\mathrm{F}^{1}$ & \multicolumn{4}{|c|}{$12,711^{*}$} & \multicolumn{4}{|c|}{$1,169^{*}$} \\
\hline
\end{tabular}

Médias seguidas da mesma letra, maiúscula na coluna e minúscula na linha, não diferem entre si, pelo teste de Tukey a $5 \% .{ }^{*}$ significativo a $5 \% .{ }^{1} \mathrm{~F}$ para interação tripla.

Para o lote de 2005 , a germinação na temperatura de $35^{\circ} \mathrm{C}$ em papel foi nula, já em areia, houve elevada germinação em todas as temperaturas testadas, que não diferiram entre si, atingindo valores de $90 \%$. Esse alto valor de germinação em areia pode ter ocorrido devido a baixa contaminação de microrganismos nesse substrato.

Para a cultivar IPR 116, verifica-se que o substrato areia propiciou maior porcentagem de germinação na primeira contagem em relação ao substrato papel, não havendo diferenças significativas entre os substratos quando se utilizou a temperatura de $20^{\circ} \mathrm{C}$ no lote de 2005 (Tabela 5). Para sementes do lote de 2004, desempenho superior foi observado na temperatura de $20^{\circ} \mathrm{C}-30^{\circ} \mathrm{C}$ em ambos os substratos e para o lote de 2005 não houve diferenças significativas entre as temperaturas em ambos os substratos.

De modo geral o substrato areia propiciou maior velocidade de germinação em relação ao substrato papel para todos os lotes e temperaturas testadas, excetuando-se nas condições de temperatura alternada, onde não foram observadas diferenças significativas nos valores de primeira contagem nos dois substratos. Resultados semelhantes relatando maior velocidade de germinação em substrato areia têm sido encontrados para várias espécies florestais, e de acordo com Pacheco et al. (2006) e Lopes et al. (2005) isso pode ser explicado pela maior área de contato da semente com a areia o que propicia maior velocidade na absorção de água.

A porcentagem de germinação (Tabela 6) foi superior no substrato areia em relação ao papel, exceto a $20^{\circ} \mathrm{C}$ para o lote de 2004 , e a $20^{\circ} \mathrm{C}-30^{\circ} \mathrm{C}$ para ambos os lotes da cultivar CATI AL-1000 quando os resultados de germinação foram superiores. Em alternância de temperatura, condições que propiciaram maior germinação das sementes, não foram observadas diferenças entre substratos indicando que quando a temperatura é adequada, o efeito do substrato no percentual de germinação das sementes de nabo forrageiro é menor. Outro aspecto a ser considerado é a maior proximidade entre as plântulas e sementes de nabo forrageiro no papel, o que poderia levantar a suspeita de aleloquímicos das plântulas afetarem a germinação do nabo forrageiro. $\mathrm{O}$ efeito de aleloquímicos de nabo forrageiro afetando a germinação de outras espécies já foi relatado por Calegari (2001) e Almeida e Rodrigues (1995).

Principalmente em substrato papel, a temperatura de $30^{\circ} \mathrm{C}$ favoreceu maior porcentagem de plântulas anormais infeccionadas e a $35^{\circ} \mathrm{C}$, maior ocorrência de sementes dormentes e plântulas deformadas (dados não apresentados) (Figura 1).

Autores como Marshall et al. (2000) observaram 
que as altas temperaturas no teste de germinação de espécies da família Brassicaceae não somente diminuem a porcentagem de plântulas normais, como também favorecem o aparecimento de sementes não germinadas
(De la Rosa- Ibarra et al., 2000). Entretanto, Momoh et al. (2002) observaram para B. napus maiores porcentagens de sementes dormentes a $20^{\circ} \mathrm{C}$ quando estas foram submetidas a uma condição de estresse.

TABELA 6. Porcentagem de germinação (\%) de sementes de nabo forrageiro das cultivares CATI AL-1000 e IPR 116 lotes de 2004 e 2005, em diferentes temperaturas e substratos sobre papel (SP) e sobre areia.

\begin{tabular}{|c|c|c|c|c|c|c|c|c|}
\hline \multirow{3}{*}{$\mathrm{T}\left({ }^{\circ} \mathrm{C}\right)$} & \multicolumn{4}{|c|}{ CATI AL-1000 } & \multicolumn{4}{|c|}{ IPR 116} \\
\hline & \multicolumn{2}{|c|}{2004} & \multicolumn{2}{|c|}{2005} & \multicolumn{2}{|c|}{2004} & \multicolumn{2}{|r|}{2005} \\
\hline & SP & Areia & SP & Areia & SP & Areia & SP & Areia \\
\hline 15 & 33 b B & 69 a A & 78 b B & 92 a A & $35 \mathrm{~b} \mathrm{BC}$ & 65 a A & $32 \mathrm{~b} A B$ & 68 a B \\
\hline 20 & 49 a $A B$ & 60 a $A B$ & $85 \mathrm{~b} \mathrm{AB}$ & 94 a A & 37 b B & 69 a A & 49 b B & 63 a B \\
\hline 25 & 39 b B & 58 a $\mathrm{AB}$ & 80 b B & 99 a A & $45 \mathrm{~b} \mathrm{AB}$ & 71 a A & $44 \mathrm{~b} \mathrm{~B}$ & $71 \mathrm{a} \mathrm{B}$ \\
\hline 30 & $11 \mathrm{~b} \mathrm{C}$ & 58 a $\mathrm{AB}$ & $37 \mathrm{~b} \mathrm{C}$ & 92 a A & $26 \mathrm{~b} \mathrm{BC}$ & 69 a A & $15 \mathrm{~b} \mathrm{BC}$ & 73 a B \\
\hline 35 & $7 \mathrm{bC}$ & 51 a B & $0 \mathrm{~b} \mathrm{D}$ & 96 a $\mathrm{A}$ & 8 b C & 68 a A & $11 \mathrm{~b} \mathrm{C}$ & 78 a $\mathrm{AB}$ \\
\hline $20-30$ & 62 a A & 62 a $A B$ & 95 a A & 97 a A & 71 a A & 74 a A & $79 \mathrm{~b} \mathrm{~A}$ & 94 a A \\
\hline Média & $34 \mathrm{~b}$ & $60 \mathrm{a}$ & $63 \mathrm{~b}$ & $95 \mathrm{a}$ & $37 \mathrm{~b}$ & $81 \mathrm{a}$ & $38 \mathrm{~b}$ & $75 \mathrm{a}$ \\
\hline $\mathrm{CV}(\%)$ & \multicolumn{2}{|c|}{14,93} & \multicolumn{2}{|c|}{4,41} & \multicolumn{2}{|c|}{15,76} & \multicolumn{2}{|c|}{10,32} \\
\hline $\mathrm{F}^{1}$ & \multicolumn{4}{|c|}{$11,459^{\mathrm{NS}}$} & \multicolumn{4}{|c|}{$1,152 *$} \\
\hline
\end{tabular}

Médias seguidas da mesma letra, maiúscula na coluna e minúscula na linha, não diferem entre si, pelo teste de Tukey a $5 \%$. * significativo a $5 \%$. ${ }^{1} \mathrm{~F}$ para interação tripla.

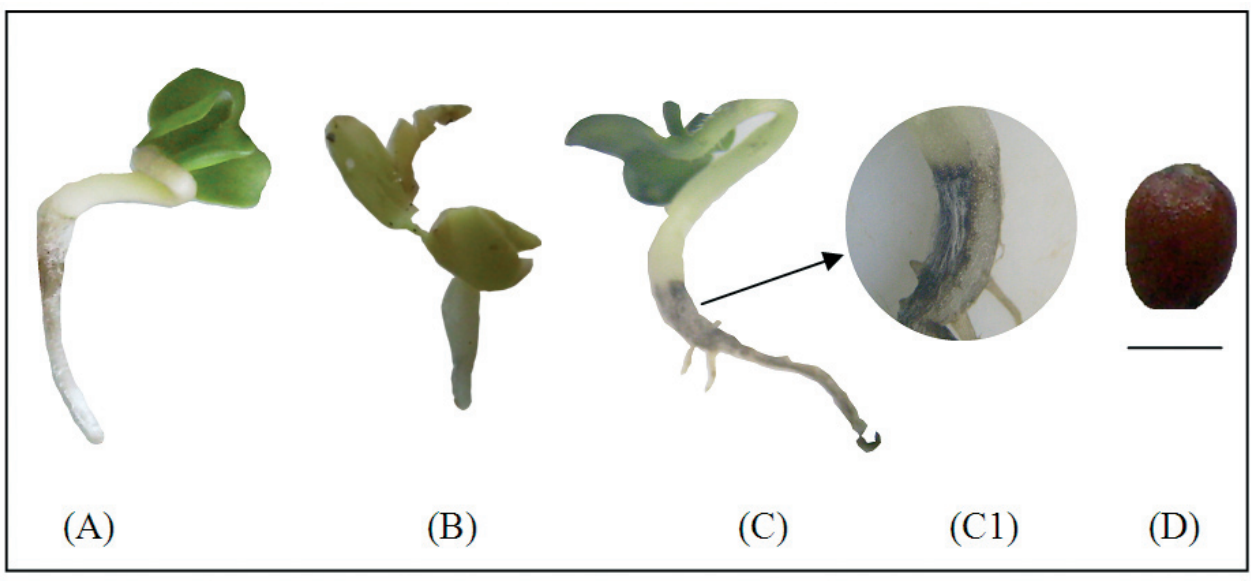

FIGURA 1. Tipo de plântulas e sementes de nabo forrageiro encontradas no teste de germinação. (A) Plântulas normais; (B) Plântulas anormais deformadas; (C) Plântulas anormais infeccionadas; (C1) Detalhe da área lesionada pela infecção e (D) Sementes dormentes. Barra $=\mathbf{2 , 0} \mathbf{~ m m}$.

Para as sementes da cultivar IPR 116, a porcentagem de germinação também foi superior no substrato areia em relação ao papel (Tabela 6), exceto para o lote de 2004 à temperatura de $20^{\circ} \mathrm{C}-30^{\circ} \mathrm{C}$, que não apresentou diferenças significativas, confirmando a hipótese do substrato não interferir na germinação do nabo forrageiro em condições ideais de temperatura, no caso a alternância de $20^{\circ} \mathrm{C}-30^{\circ} \mathrm{C}$. No entanto, o substrato areia propiciou, para o lote de 2004, germinação semelhante independente das temperaturas utilizadas. em substrato papel, nas temperaturas de $30^{\circ} \mathrm{c}$ e 
$35^{\circ} \mathrm{C}$, foram observadas maiores porcentagens de plântulas com danos (dados não apresentados), sendo que a $35^{\circ} \mathrm{C}$ houve maior porcentagem de sementes mortas.

As altas temperaturas podem ser deletérias para geminação de sementes, pois temperaturas acima da ótima podem causar efeitos sobre a atividade de enzimas, além de reduzir a emergência (Nascimento e Cantliffe, 2002; Wahid et al., 2007). Outras espécies da família Brassicaceae também têm a germinação das sementes afetada pelas altas temperaturas, como B. napus L., canola (Young et al., 2004) e $B$. juncea 1., mostarda (Rao et al., 1992). Blackshaw et al. (1991) relatam que a germinação de sementes de B. napus foi reduzida quando as sementes foram postas para germinar a $30^{\circ} \mathrm{C}$. Entretanto, B. campestri teve sua germinação favorecida a $30^{\circ} \mathrm{C}$ (De la Rosa-Ibarra et al., 2000), o que indica resposta diferencial da temperatura na germinação.

O índice de velocidade de germinação das sementes de nabo forrageiro foi superior em substrato areia em relação ao substrato papel, exceto para temperatura alternada de $20^{\circ} \mathrm{C}-30^{\circ} \mathrm{C}$ para a cultivar CATI AL-1000. O IVG superior foi observado na temperatura de $20^{\circ} \mathrm{C}-30^{\circ} \mathrm{C}$ em substrato papel no lote de 2004 e 2005 , e também a $25^{\circ} \mathrm{C}$ em substrato areia (Tabela 7).

TABELA 7. Resultado do índice de velocidade de germi- -nação (IVG) de sementes de nabo forrageiro das cultivares CATI AL-1000 e IPR 116 lotes de 2004 e 2005, em função de diferentes temperaturas e substratos sobre papel (SP) e sobre areia.

\begin{tabular}{|c|c|c|c|c|c|c|c|c|}
\hline \multirow{3}{*}{$\mathrm{T}\left({ }^{\circ} \mathrm{C}\right)$} & \multicolumn{4}{|c|}{ CATI AL-1000 } & \multicolumn{4}{|c|}{ IPR 116} \\
\hline & \multicolumn{2}{|c|}{2004} & \multicolumn{2}{|c|}{2005} & \multicolumn{2}{|c|}{2004} & \multicolumn{2}{|r|}{2005} \\
\hline & SP & Areia & SP & Areia & SP & Areia & SP & Areia \\
\hline 15 & $8 \mathrm{~b} \mathrm{BC}$ & 18 a $\mathrm{A}$ & $18 \mathrm{~b} \mathrm{~A}$ & 29 a $\mathrm{AB}$ & $7 \mathrm{~b} \mathrm{BC}$ & 14 a $\mathrm{A}$ & $10 \mathrm{bBCD}$ & $19 \mathrm{a} A B$ \\
\hline 20 & $11 \mathrm{~b} \mathrm{AB}$ & 15 a $\mathrm{A}$ & $21 \mathrm{~b} \mathrm{~A}$ & 27 a B & $7 \mathrm{~b} \mathrm{BC}$ & 17 a A & $13 \mathrm{bABC}$ & 18 a B \\
\hline 25 & $10 \mathrm{~b} \mathrm{AB}$ & 15 a $\mathrm{A}$ & $21 \mathrm{~b} \mathrm{~A}$ & 28 a A & $9 \mathrm{~b} \mathrm{~B}$ & 17 a $\mathrm{A}$ & $14 \mathrm{~b} \mathrm{AB}$ & $20 \mathrm{a} \mathrm{AB}$ \\
\hline 30 & $7 \mathrm{~b} \mathrm{BC}$ & 15 a $\mathrm{A}$ & $20 \mathrm{~b} \mathrm{~B}$ & 32 a $\mathrm{AB}$ & $9 \mathrm{~b} \mathrm{BC}$ & 17 a $\mathrm{A}$ & $9 \mathrm{~b} \mathrm{CD}$ & $20 \mathrm{a} \mathrm{AB}$ \\
\hline 35 & 8 b C & 15 a $A$ & $6 \mathrm{~b} \mathrm{~B}$ & 27 a B & 4 b C & 17 a $\mathrm{A}$ & 6 b D & $22 \mathrm{a} \mathrm{AB}$ \\
\hline $20-30$ & 15 a A & 16 a A & $24 \mathrm{a} \mathrm{A}$ & 28 a AB & 16 a A & 18 a A & $16 \mathrm{~b} \mathrm{~A}$ & 24 a A \\
\hline Média & $10 \mathrm{~B}$ & $16 \mathrm{~A}$ & $18 \mathrm{~B}$ & $29 \mathrm{~A}$ & $9 \mathrm{~B}$ & $17 \mathrm{~A}$ & $11 \mathrm{~B}$ & $21 \mathrm{~A}$ \\
\hline $\mathrm{CV}(\%)$ & \multicolumn{2}{|c|}{10,74} & \multicolumn{2}{|c|}{7,51} & \multicolumn{2}{|c|}{12,14} & \multicolumn{2}{|c|}{8,78} \\
\hline $\mathrm{F}^{1}$ & \multicolumn{4}{|c|}{$1,213^{\mathrm{NS}}$} & \multicolumn{4}{|c|}{$3,155^{*}$} \\
\hline
\end{tabular}

Médias seguidas da mesma letra, maiúscula na coluna e minúscula na linha, não diferem entre si, pelo teste de Tukey a $5 \% . *$ significativo a $5 \% .{ }^{1} \mathrm{~F}$ para interação tripla.

Para a cultivar IPR 116, IVG superior foi obtido em substrato areia, exceto para o lote de 2004 a temperatura de $20^{\circ} \mathrm{C}-30^{\circ} \mathrm{C}$ que não apresentou diferenças significativas entre os substratos (Tabela 7). A temperatura de $20^{\circ} \mathrm{C}$ $30^{\circ} \mathrm{C}$ também promoveu resultados superiores de IVG em substrato areia no lote de 2005.

Os substratos testados nesse estudo influenciaram a germinação das sementes de nabo forrageiro, uma vez que, em substrato areia houve maior velocidade de germinação e, com isso, a possibilidade de escape das sementes aos fungos que afetam as sementes em início de germinação. Além disso, em substrato papel de filtro em caixas gerbox, ocorreu desidratação rápida, excessiva e desigual, sendo necessário o reumedecimento no decorrer do teste. É importante salientar, que quando se utilizou quantidade de água equivalente a 2,5 vezes o peso do papel para o umedecimento deste, conforme recomendado por Brasil (1992), as sementes se deterioraram devido à maior incidência de fungos sendo, portanto, utilizado 2,0 vezes o peso do papel.

Segundo Andrade et al. (2006), a utilização de substrato sobre papel proporciona menores médias de germinação e vigor, já que promove menor capacidade de retenção de água. 
A avaliação do número de sementes germinadas em dias consecutivos permitiu a confecção das curvas de germinação acumulativas para a cultivar CATI AL-1000 (Figura 2) e para a cultivar IPR 116 (Figura 3). Observase uma distribuição temporal da germinação, com um padrão normal ou gaussiano. Este mesmo modelo pode ser observado para a germinação de sementes de B. napus L. (Marshall et al., 2000), B. rapa L., nabo (Gong et al., 2001) e também para B. campestri L., colza (Sinniah et al., 1998). Verificou-se que a partir do $3^{\circ}$ dia de germinação já haviam plântulas normais que pudessem ser retiradas do substrato, sendo este período adotado como a data da primeira contagem, já que os apresentaram mais de $50 \%$ das sementes germinadas. Ao $4^{\circ}$ dia, as curvas de germinação começaram a se estabilizar. $\mathrm{O}$ último dia do teste foi delimitado ao $7^{\circ}$ dia, quando já não foi possível observar o aparecimento de novas plântulas, restando apenas sementes dormentes e mortas. Comportamento diferenciado entre os tratamentos, com menor sincronismo, foi verificado à temperatura de $35^{\circ} \mathrm{C}$, tendendo a ser semelhante na temperatura de $20^{\circ} \mathrm{C}-30^{\circ} \mathrm{C}$ em ambos os substratos.
(1)

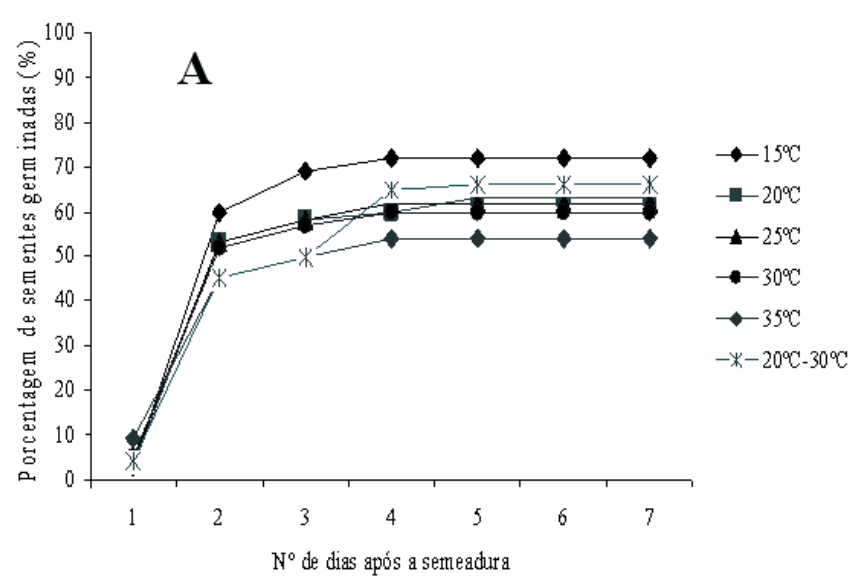

(2)

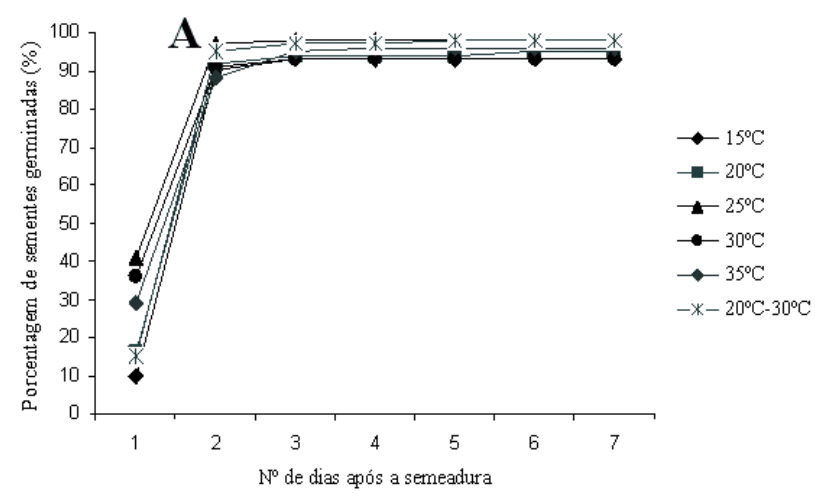

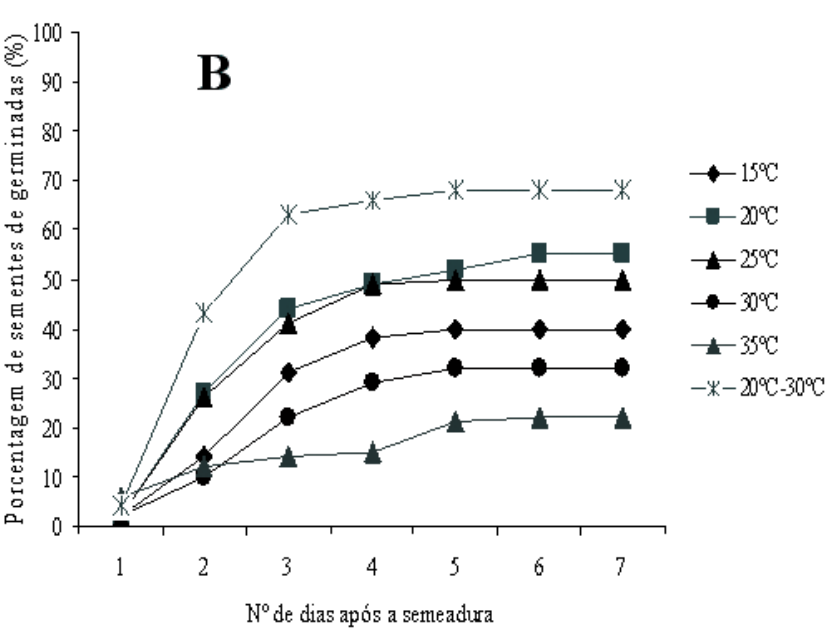

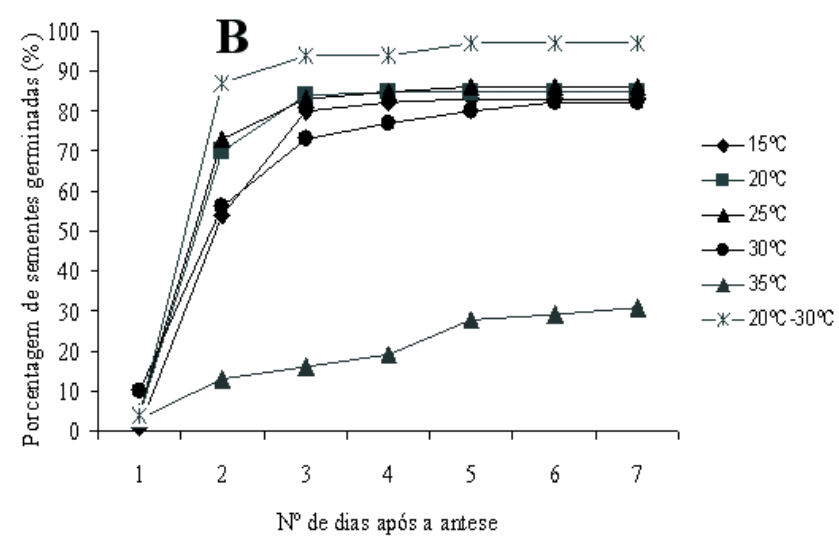

FIGURA 2. Curvas de germinação (\%) acumulada de sementes de nabo forrageiro cultivar CATI AL-1000 lote de 2004 (1) e 2005 (2), nas diferentes temperaturas e em substrato areia (A) e sobre papel (B). 

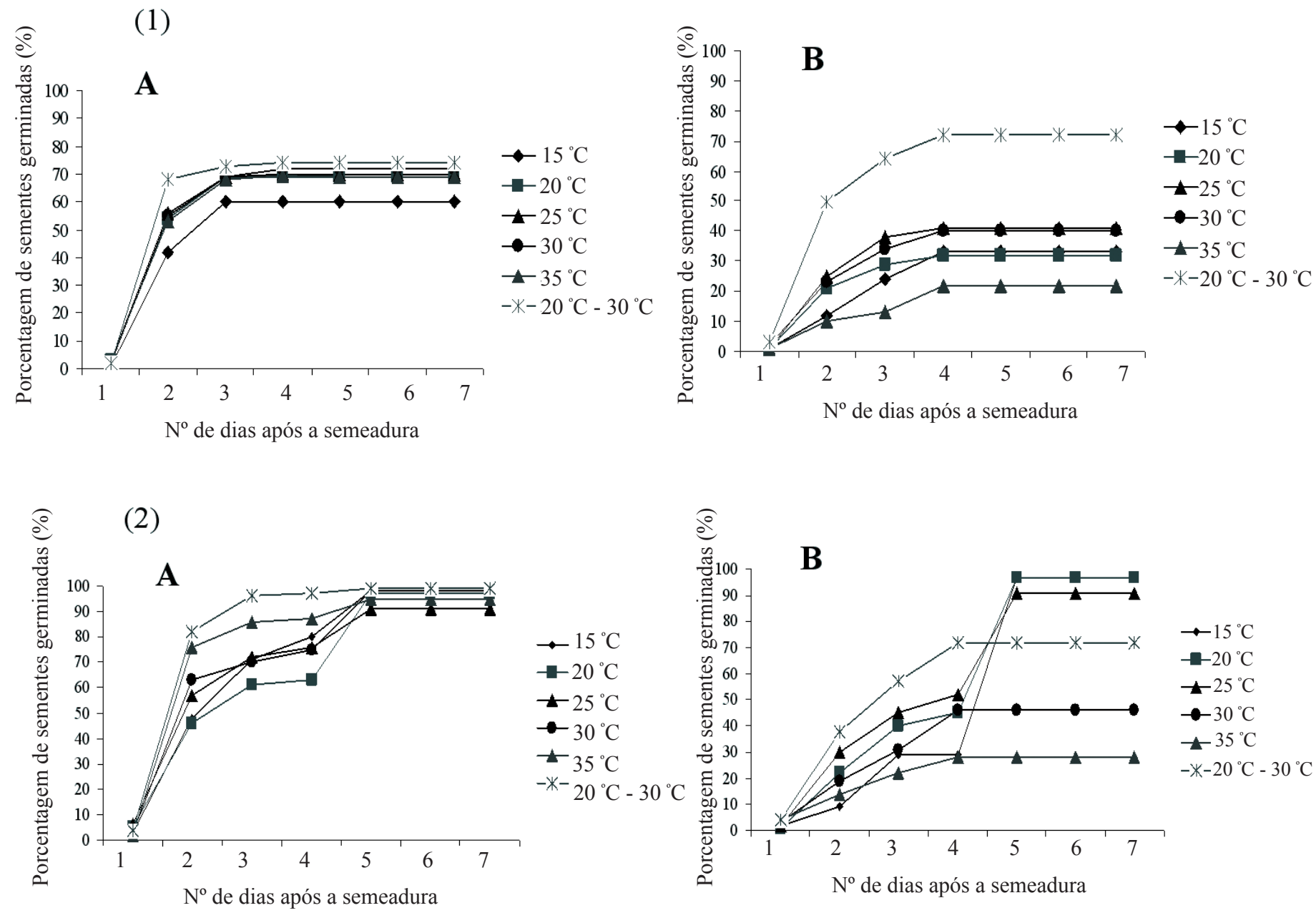

FIGURA 3. Curvas de germinação (\%) acumulada de sementes de nabo forrageiro cultivar IPR 116 lote de 2004 (1) e 2005 (2), nas diferentes temperaturas e em substrato areia (A) e sobre papel (B).

\section{CONCLUSÃO}

A temperatura de $20^{\circ} \mathrm{C}-30^{\circ} \mathrm{C}$ e o uso do substrato areia são condições adequadas para avaliação da germinação das sementes de nabo forrageiro das cultivares CATI AL-1000 e IPR 116.

\section{AGRADECIMENTOS}

A FAPEMIG e CNPq pelo apoio financeiro.

\section{REFERÊNCIAS}

ADAM, N. R.; DIERIG, D. A.; COFFELT, T. A.; WINTERMEYER, M. J.; MACKEY, B. E.; WALL, G. W. Cardinal temperatures for germination and early growth of two Lesquerella species. Industrial Crops and Products, v. 25, n. 1, p. 24-33, Jan. 2007.

AGUIAR, I. B.; PIÑA-RODRIGUES, F. C. M.; FIGLIOLIA, M. B. Sementes florestais tropicais. Brasília, DF: ABRATES, 1993. 350 p.

ALMEIDA, F. S.; RODRIGUES, B. N. Guia de herbicidas: recomendações para o uso adequado em plantio direto e convencional. Londrina: IAPAR, 1995. 482.

ALVINO, F. O.; RAYOL, B. P. Efeito de diferentes substratos na germinação de Ochroma pyramidale (CAV. EX LAM.) URB. (Bombacaceae). Ciência Florestal, v. 17, n. 1, p. 71-75, jan./mar. 2007.

ANDRADE, A. C. S.; PEREIRA, T. S.; FERNANDES, M. J.; CRUZ, A. P. M.; CARVALHO, A. S. R. Substrato, 
temperatura de germinação e desenvolvimento pós-seminal de sementes de Dalbergia nigra. Pesquisa Agropecuária Brasileira, v. 41, n. 3, p. 517-523, mar. 2006.

BERTI, M. T.; JOHNSON, B. L.; MANTHEY, L. K. Seed physiological maturity in cuphea. Industrial Crops and Products, v. 25, n. 2, p. 190-201, Feb. 2007.

BEWLEY, J. D.; BLACK, M. Seeds: physiology of development and germination. 2. ed. New York: Plenum, 1994. 445 p.

BLACKSHAW, R. E., Soil temperature and moisture effects on downy brome vs. winter canola, wheat, and rye emergence. Crop Science, v. 31, n. 4, p. 1034-1040, July/Aug. 1991.

BRASIL. Ministério da Agricultura e Reforma Agrária. Regras para análise de sementes. Brasília, DF: SNDA/ DNDV/CLAV, 1992. 365 p.

BURRELL, N. J.; KNIGHT, G. P.; ARMITAGE, D. M.; HILL, S. T. Determination of the time available for drying rapeseed before the appearance of surface moulds. Journal of Stored Products Research, v. 16, n. 3-4, p. 115-118, Dec. 1980.

CALEGARI, A. Sustentabilidade sim. In: ENCONTRO DE PLANTIO DIRETO NO CERRADO, 5., 2001, Dourados. Anais... Dourados: UFMS/Embrapa Agropecuária Oeste, 2001. p. 23-28.

CHAUHAN, B. S.; GILL, G.; PRESTON, C. African mustard (Brassica tournefortii) germination in southern Australia. Weed Science, v. 54, n. 5, p. 891-897, Sept./Oct. 2006.

CHEN, C.; JACKSON, G.; NEILL, K.; WICHMAN, D.; JOHNSON, G.; JOHNSON, D. Determining the Feasibility of Early Seeding Canola in the Northern Great Plains. Agronomy Journal, v. 97, n. 4, p. 1252-1262, July/Aug. 2005.

\section{COMMERCIAL AGRICULTURE DEVELOPMENT.}

Procedures for the wet towel germination test. Fairbanks: University of Alaska Fairbanks Cooperative Extension Service programs. Disponível em: $<\mathrm{http} / / \mathrm{www}$.uaf.edu/ces $>$. Acesso em: 01 set. 2006.

DE LA ROSA-IBARRA, M.; MAITI, R. K.; GARZASAENZ, O. Germination and methods to break seed dormancy of Brassica juncea and B-campestris. Phyton-International Journal of Experimental Botany, v. 66, p. 93-96, 2000.

DERPSCH, R.; CALEGARI, A. Plantas para adubação verde de inverno. 2.ed. Londrina: IAPAR, 1992. p. 1-78
(IAPAR. Circular, 73).

ELLIS, R. H.; HONG, T. D.; ROBERTS, E. H. Quantal response of seed germination in seven genera of Cruciferae to white light of varying photon flux density and photoperiod. Annals of Botany, London, v. 63, n. 1, p. 145-158, 1989.

FANTI, S. C.; PEREZ, S. C. J. Influência do substrato e do envelhecimento acelerado na germinação de olho-de-dragão (Adenanthera pavonina L. - Fabaceae). Revista Brasileira de Sementes, v. 2, n. 2, p. 135-141, 1999.

FERREIRA, D. F. SISVAR-Sistema de análise de variância para dados balanceados: programa de análises estatísticas e planejamento de experimentos, versão 4.1. Lavras: UFLA, 2000 .

FINCH-SAVAGE, W. E.; Leubner- METZGER, G. L. Seed dormancy and the control of germination. New Phytologist, v. 171, n. 3, p. 501-523, 2006.

GONG, P.; WIKE, B. M.; STROZZI, E.; FLEISCHAMANN, S. Evaluation and refinement of a continuous seed germination and early seedling growth test for the use in the ecotoxicological assessment of soils. Chemosphere, v. 44, n. 3, p. 491-500, July 2001.

HUMPHERSON-JONES, F. M. Epidemiology and control of dark leaf spot of brassicas. In: CHELKOWSKI, J.; VISCONTI, A. (Ed.). Alternaria: biology, plant diseases and metabolites. Amsterdam: Elsevier, 1992. p. 267-288.

KOGER, C. H.; REDDY, K. N.; POSTON, D. H. Factors affecting seed germination, seedling emergence, and survival of texasweed (Caperonia palustris). Weed Science, v. 52, n. 6, p. 989-995, Nov. 2004.

LOPES, J. C.; CAPUCHO, M. T.; MARTINS FILHO, S.; REPOSSI, P. A. Influência de temperatura, substrato e luz na germinação de sementes de bertalha. Revista Brasileira de Sementes, v. 27, n. 2, p. 18-24, dez. 2005.

MAGUIRE, J. D. Speed of germination: aid in selection and evaluation for seedling emergence and vigor. Crop Science, Madison, v. 2, n. 2, p. 176-177, Mar./Apr. 1962.

MARSHALL, B.; DUNLOP, G.; RAMSAY, G.; SQUIRE, G. R. Temperature-dependent germination traits in oilseed rape associated with $5^{\prime}$-anchored simple sequence repeat PCR polymorphisms. Journal of Experimental Botany, v. 51, n. 353, p. 2075-2084, Dec. 2000.

MAUDE, R. B.; HUMPHERSON-JONES, F. M. Studies on the seed-borne phases of dark leaf spot (Alternaria 
brassicicola) and grey leaf spot (Alternaria brassicae) of brassicas. Annals of Applied Biology, n. 95, n. 3, p. 311319, 1980.

MOMOH, E. J. J.; ZHOU, W. J.;KRISTIANSSON, B. Variation in the development of secondary dormancy in oilseed rape genotypes under conditions of stress. European Weed Research Society, Weed Research, v. 42, n. 6, p.446455, Dec. 2002.

MUZILLI, O. Manejo da matéria orgânica no sistema plantio direto: a experiência no Estado do Paraná. Informações Agronômicas, Piracicaba, n. 100, p. 6-10, dez. 2002.

NASCIMENTO, W. M.; CANTLIFFE, D. J. Germinação de sementes de alface sob altas temperaturas. Horticultura Brasileira, v. 20, n. 1, p.103-106, mar. 2002.

OLIVEIRA, A. P.; BRUNO, R. L. A.; ALVES, E. U. Influência do substrato e da temperatura na germinação de sementes peletizadas de tomate. Revista Brasileira de Sementes, v. 23, n. 2, p.72-77, 2001.

PACHECO, M. V.; MATOS, V. P.; FERREIRA, R. L. C.; FELICIANO, A. L. P.; PINTO, K. M. S. Efeito de temperaturas e substratos na germinação de sementes de Myracrodruon urundeuva Fr. All. (Anacardiaceae). Revista Árvore, v. 30, n. 3, p. 359-367, maio/jun. 2006.

PEREIRA, R. S.; MUNIZ, M. F. B.; NASCIMENTO, W. M. Aspectos relacionados à qualidade de sementes de coentro. Horticultura Brasileira, v. 23, n. 3, p. 703-706, jul./set. 2005.

PRONYK, C.; ABRAMSONB, D.; MUIR, W. E; WHITE, N. D. G. Correlation of total ergosterol levels in stored canola with fungal deterioration. Journal of Stored Products Research, v. 42, n. 2, p.162-172, 2006.

RAO, G. U.; JAIN, A.; SHIVANNA, K. R. Effects of high temperature stress on Brassica pollen: viability, germination and ability to set fruits and seeds. Annals of Botany, v. 69, n. 3, p. 193-198, Mar. 1992.

SHRESTHA, S. K.; MATHUR, S. B.; MUNK, L. Alternaria brassicae in seeds of rapeseed and mustard, its location in seeds, transmission from seeds to seedlings and control. Seed Science and Technology, v. 28, n. 1, p.75-84, 2000.

SILVA, A. R. B.; SILVA, T. R. B.; SILVA, M. L. L.; VIANNA, J. F.; MARTINEZ, M. M.; VIANAS, L. H.; SILVA, R. F. Comportamento de cultivares de nabo forrageiro (Raphanus sativus $L$.) em função da variação do espaçamento entre linhas. Disponível em: $<$ http//www. portaldobiodiesel. gov.br>. Acesso em: 15 jan. 2007.

SIMPSON, G. G.; DEAN, C. Arabidopsis, the Rosetta Stone of Flowering Time? Science, v. 296, n. 5566, p. 285 - 289, Apr. 2002.

SINNIAH, U. R.; ELLIS, R. H.; JOHN, P. Irrigation and Seed Quality Development in Rapid-cycling Brassica: Seed Germination and Longevity. Annals of Botany, v. 82, n. 3, p.309-314, Sept. 1998.

SQUIRE, G. R.; MARSHALL, B.; DUNLOP, G.; WRIGHT, G. Genetic basis of rate-temperature characteristics forgermination in oilseed rape. Journal of Experimental Botany, v. 48, n. 309, p. 869-875, Apr. 1997.

SZOPIŃSKA, D.; TYLKOWSKA, K.; STACH, A. Relationships between seed development stage, germination, occurrence and location of fungi in oilseed rape (Brassica napus ssp. oleifera L.) seeds and the presence of Alternaria AND Cladosporium spp. spores in the air. Electronic Journal of Polish Agricultural Universities, v. 10, n. 4, p. 19. 2007.

TOBE, K.; ZHANG, L.; OMASA, K. Seed germination and seedling emergence of three annuals growing on desert sand dunes in China. Annals of Botany, v. 95, n. 4, p. 649-659, Mar. 2005.

TOKUMASU, S.; KAKIHARA, F. Seasonal germination periodicity of imbibed dormant seeds of rape (Brassica napus L.). Scientia Horticulturae, v. 42, n. 1-2, p. 1-7, Mar. 1990.

WAHID, A.; GELANI, S.; ASHRAF, M.; FOOLAD, M. R. Heat tolerance in plants: An overview. Environmental and Experimental Botany, v. 61, n. 3, p. 199-223, Dec. 2007.

YOUNG, L. W.; WILEN, R. W.; BONHAM-SMITH. High temperature stress of Brassica napus during flowering reduces micro- and megagametophyte fertility, induces fruit abortion, and disrupts seed production. Journal of Experimental Botany, v. 55, n. 396, p. 485-495, Feb. 2004.

PROBERT, R.J.; SMITH, R.D.; BIRCH, P. Germination responses to light and alternating temperatures in European populations of Dactylis glomerata L.V. The principal components of the alternating temperature requirements. News Phytologist, v.102, p. 133-142, 1986. 